\title{
Mycobacterium thermoresistibile
}

National Cancer Institute

\section{Source}

National Cancer Institute. Mycobacterium thermoresistibile. NCI Thesaurus. Code C86593.

A species of aerobic, Gram-positive, rod shaped bacteria assigned to the phylum Actinobacteria. This species is acid-fast, rapidly growing, catalase, urease and Tween 80 hydrolysis positive, and tellurite reductase, iron uptake, and arylsulfatase (14 days) neg ative, and is susceptible to ciprofloxacin. M. thermoresistibile is found in soil and is strongly associated with pulmonary and skin infections, but rarely isolated from clinical samples. 